Rheumatoid vasculitis (RV) is an extra-articular manifestation of rare but serious rheumatoid disease that involves the damage of small and medium sized vessels.

Objectives: The purpose of the study was to evaluate the incidence of damages occurred on cutaneous level in patients diagnosed with RA and to detect capillaroscopic microangiopathy changes in the activity of rheumatoid disease. Methods: 101 patients were included in the study, all were diagnosed with RA according to the ACR/EULAR 2010 criteria. RV diagnosis was based on the clinical examination. The capillaroscopic evaluation was performed with a 3.0 VideoCap device at a 200x magnification (200x magnification contact lenses). Capillaroscopic examination was performed on fingers II-V of both hands, being considered pathological if the capillaroscopic changes are present in at least two fingers. The activity of disease was calculated using DAS 28 ESR score.

Results: $18 \%$ of examined patients was male and $82 \%$ female, with the mean age of 56,2 years $\pm 10.16 S D$, and the mean duration of the disease in years of illness was $8.65 \pm 5.31$ SD. 3 patients had periungual vasculitis with cutaneous ulceration and gangrene, 12 patients had palpable purpura. $29 \%$ of the patients had vasospastic skin changes. The activity score of RA was associated with the capillaroscopic changes in peripheral microangiopathy $p-0.037$. Capillaries images were heterogenous in aspects and distribution, tortuous capillaries, microhemorrhages, giant/dilated capillaries, avascular areas were observed.

Conclusion: Vascular microangiopathy evidenced by capillaroscopic examination is present in patients with cutaneous vasculitis having a medium, severe activity of rheumatoid diseases.

References:

[1] Cutolo M, Paolino S, Smith V. Nailfold capillaroscopy in rheumatology: ready for daily use but care in terminology. Clinical Rheumatology 2019; 38:22932297 http://doi.org/10/s10067-019-04716-w

[2] Lambova N, Müller-Ladner U. Capillaroscopic pattern in inflammatory arthritis. Microvascular Research 2012; 83:318-322
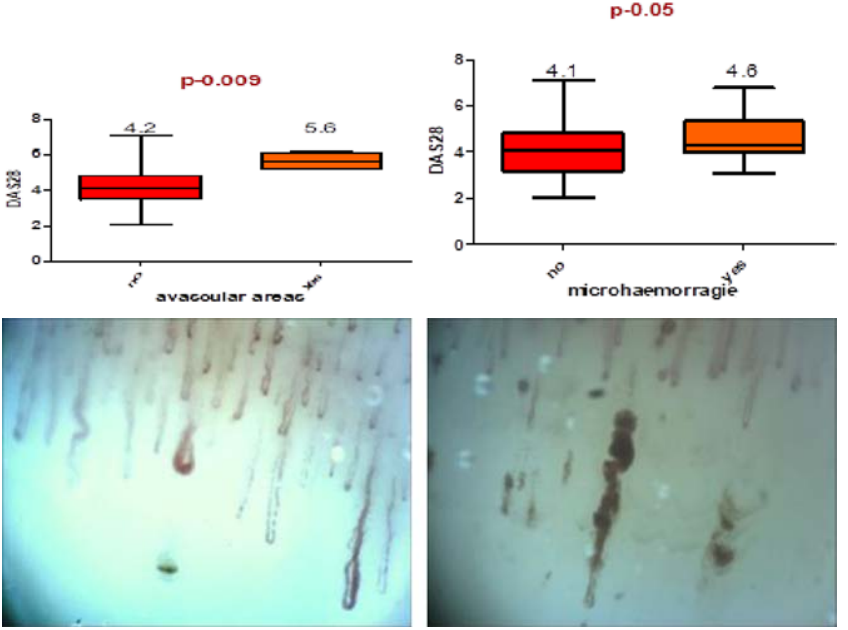

Dilated, giant capillaries, microhemorrhage bleedings, avascular area Reduced blood flow (observed in dynamics)

Disclosure of Interests: Mirela Parvu Speakers bureau: Not for this subject, theme, Anca Cozos Speakers bureau: Not for this subject, theme, Anna-Iuliana Biro Speakers bureau: Not for this subject, theme, Horatiu Valeriu Popoviciu Speakers bureau: Not for this subject, theme DOI: 10.1136/annrheumdis-2020-eular.6150

\section{AB0260 $\quad$ SARCOPENIA AND RHEUMATOID ARTHRITIS}

V. Povoroznyuk ${ }^{1}$, N. Dzerovych ${ }^{1}$, O. Ivanyk ${ }^{1}$, T. Karasevska ${ }^{2} .{ }^{1}$ D. F. Chebotarev Institute of Gerontology NAMS of Ukraine, Kyiv, Ukraine; ${ }^{2} \mathrm{O}$. Bogomolets National Medical University, Kyiv, Ukraine

Background: Nowadays in the field of syndromes and diseases associated with age, scientists focus especial attention on the problem of sarcopenia, which combines an increased risk of falls, deterioration of life quality, impaired functional activity, reduced life expectancy and increased mortality of patients. In 2016, sarcopenia has been included in the International Classification of Diseases. There are the primary and secondary forms of sarcopenia.

Objectives: The aim of this study was to evaluate the bone mineral density, lean mass, frequency of pre-sarcopenia and analyze correlation among the activity parameters, duration of the disease, life quality and lean mass indices in women with rheumatoid arthritis.

Methods: 461 women aged $40-87$ years (age $-57.17 \pm 0.71$ years) were exam ined, among them 71 patients with rheumatoid arthritis and 390 controls. We conducted the clinical and laboratory examination. Pain intensity was evaluated by the visual analogue scale, the quality of life - by the HAQ questionnaire. Lean mass, bone mineral density were measured by the X-ray absorptiometry (Prodigy, GEHC Lunar, Madison, WI, USA). Pre-sarcopenia was determined when an appendicula lean mass index was less than $5.72 \mathrm{~kg} / \mathrm{m} 2$ (V. Povoroznyuk, N. Dzerovych, 2016). Results: Patients with rheumatoid arthritis had a significantly lower femoral neck mineral density $(p=0.002)$, lean mass of the total body $(p=0.01)$ and appendicular lean mass $(p<0.01)$. We didn't find any significant connection among the activity parameters (C-reactive protein, ESR, pain VAS, DAS-28), duration of the disease, life quality and lean mass indices in patients with rheumatoid arthritis. However, a significant correlation was found between the number of swollen joints and lean mass of upper limbs $(r=0.67 ; p=0.02)$. The frequency of pre-sarcopenia in women with rheumatoid arthritis was $49 \%$, in the control group - $18 \%$. Conclusion: Patients with rheumatoid arthritis had not only bone tissue, but also skeletal muscle tissue disorders, resulting in a significant deterioration of functional capacity and quality of life. Given the significant medical and social significance of the problem, further studies into the mechanisms of pathogenesis, development of diagnostic methods, prevention and treatment of sarcopenia in patients with rheumatoid arthritis are required.

Disclosure of Interests: None declared DOI: 10.1136/annrheumdis-2020-eular.2727

\section{AB0261 IMPACT OF COMORBIDITIES IN THE DISEASE ACTIVITY OF PATIENTS WITH SPONDYLOARTHRITIS AND RHEUMATOID ARTHRITIS: TUNISIAN REGISTRY (BINAR)}

R. Tekaya ${ }^{1}$, L. Rouached ${ }^{1}$, A. Ben Tekaya ${ }^{1}$, O. Saidane ${ }^{1}$, S. Bouden ${ }^{1}$, I. Mahmoud ${ }^{1}$, S. Baklouti ${ }^{2}$, N. Bergaoui ${ }^{3}$, E. Bouajina ${ }^{4}$, M. Elleuch ${ }^{5}$, I. Gharsallah ${ }^{6}$, M. M. Kchir ${ }^{7}$, S. Kochbati ${ }^{8}$, A. Laatar ${ }^{9}$, Y. Mohamed ${ }^{10}$,

L. Abdelmoula ${ }^{1}$. ${ }^{1} \mathrm{Hospital}$ Charles Nicolle, Rheumatology, Tunis, Tunisia; ${ }^{2} \mathrm{Hedi}$ Chaker Hospital, Rheumatology, Sfax, Tunisia; ${ }^{3}$ Fattouma Bourguiba Hospital, Rheumatology, Monastir, Tunisia; ${ }^{4}$ Farhat Hached hospital, Rheumatology, Sousse, Tunisia; ${ }^{5}$ La Rabta, Rheumatology, Tunis, Tunisia; ${ }^{6}$ The Military Hospital in Tunis, Rheumatology, Tunis, Tunisia; ${ }^{7}$ Mohamed Kassab Institut, Rheumatology, Manouba, Tunisia; ${ }^{8} \mathrm{Hôpital} \mathrm{Habib} \mathrm{Thameur,} \mathrm{Rheumatology,}$ Tunis, Tunisia; ${ }^{9} \mathrm{Hop}$ ital Mongi Slim, Rheumatology, Marsa, Tunisia; ${ }^{10} \mathrm{Hospital}$ University Tahar Sfar, Rheumatology, Mahdia, Tunisia

Background: Comorbidities can be associated with rheumatoid arthritis (RA) and spondyloarthritis (SpA). This association can be fortuitous but can also be secondary to rheumatism itself or to the effects of the treatments used. These comorbidities can worsen the disease and even increase patient mortality.

Objectives: To assess the prevalence of comorbidities in RA or SpA patients from the Tunisian Blologics National Registry (BINAR) and to focus on their influence on the disease activity.

Methods: BINAR is a multicenter non-interventional and prospective study, conducted in Tunisia with 80 rheumatologists over a period of three years. It included patients with RA (ACR / EULAR 2010 criteria) or SpA (ASAS 2009 criteria). Data were collected and analyzed through an electronic platform managed by DACIMA. They included demographic data, smoking status and types of comorbidities (cardiovascular disease, diabetes, dyslipidemia, osteoporosis, high blood pressure (HBP), neoplasia, gastrointestinal ulcer, depression and fibromyalgia). RA activity was evaluated by the DAS28-VS score and SpA activity by the BASDAI and ASDAS-CRP scores.

Results: We included 298 patients (175 PR and $123 \mathrm{SpA}$ ) making the mean sex ratio 0.6 and mean age 49.18 years \pm 14.1 [18-79]. Mean $B M I$ was $27.0 \pm 5.5 \mathrm{~kg}$ $\mathrm{m}^{2}[15-45]$ and $17.7 \%$ of the patients were current smokers. Concerning disease activity, mean DAS28-VS in RA was at $4.9 \pm 1.5$ [1.1 - 8.1 and mean BASDAl and ASDAS-CRP, in SpA, were respectively $4.1 \pm 1.8$ and $2.8 \pm 1.1$. Comorbidities were noted in $54 \%$ of patients $(62.1 \%$ in SpA and $37.9 \%$ in RA), with an average of 1.7 comorbidities per patient.

The most common comorbidities were osteoporosis $(38.8 \%)$, cardiovascular disease $(20.1 \%)$, diabetes $(16.8 \%)$, HBP $(18.1 \%)$, dyslipidemia $(6.7 \%)$ and GIU $(6.0 \%)$. Depression, fibromyalgia and neoplasia were mentioned in $1.7 \%, 1 \%$ and $1 \%$, respectively.

No correlation was found between the number of comorbidities and the activity level of RA: DAS28-VS ( $p=0.12)$, nor the activity level of SpA: BASDAI $(p=0.07)$ ASDAS-CRP $(p=0.15)$. Correlations were studied between each comorbidity and activity disease parameters of RA and SpA, they are specified in Table 1. We found that only the presence of osteoporosis was associated with SpA activity, (ASDAS-CRP; $p=0.02$ ). 